\title{
Auswirkungen auf die Mobilitätswahrnehmung durch die Einführung eines autonomen Shuttlebusses
}

\author{
Anja Baniewicz und Christian Neff
}

Der öffentliche Personennahverkehr (ÖPNV) befindet sich derzeit in einem starken Wandel, der bestimmt wird durch Schlagworte wie Mobilität 4.0, Digitalisierung oder eben auch Autonomer ÖPNV. Das nachfolgende Kapitel behandelt die Frage, ob die Integration eines autonomen Shuttlebusses in die Angebotsstruktur des ÖPNV im ländlichen Raum dazu beitragen kann, die Mobilitätsoptionen der dortigen Bevölkerung sinnvoll zu erweitern. Die Autoren nehmen dabei die Sicht des ÖPNV-Kunden im ländlichen Raum ein und legen dar, inwieweit die Einführung und der Betrieb eines autonomen Shuttlebusses dazu beitragen, dass Mobilität im ländlichen Raum stärker resp. auf andere Weise wahrgenommen wird. Da Mobilitätswahrnehmung stets individuell geschieht, versuchen die Autoren, Indikatoren auf Grundlage zweier Haushaltsbefragungen zu konstruieren, die Aussagen über eine veränderte Mobilitätswahrnehmung ermöglichen.

\subsection{Mobilitätswahrnehmung im ländlichen Raum}

Die Auswirkungen des demografischen Wandels auf ländliche Regionen lassen sich bereits seit mehreren Jahren beobachten: Die Abnahme der Bevölkerung aufgrund von Migration in wirtschaftsstarke Regionen führt in vielen ländlichen Räumen zu einer Überalterung der Bevölkerung (BMEL 2016, S. 10). Der Bevölkerungsschwund wird in ländlichen Regionen dementsprechend von einer erhöhten Altersstruktur und einer Abnahme wohnortnaher Versorgungseinrichtungen begleitet. Vor diesem Hintergrund sind der Zugang zu Mobilität und deren Erhalt in ländlichen Räumen entscheidende Faktoren für die Lebensqualität und tragen somit dazu bei, dass ländliche Räume auch zukünftig als attraktive

\footnotetext{
A. Baniewicz $\cdot$ C. Neff $(\square)$

DB Regio Bus Region Bayern, Ingolstadt, Deutschland

E-Mail: anja.baniewicz@ deutschebahn.com; christian.neff@ deutschebahn.com
} 
Wohn-, Lebens- und Erholungsräume wahrgenommen werden. Die Möglichkeit, mobil zu sein, stellt gerade im ländlichen Raum eine zentrale Voraussetzung dar, um am gesellschaftlichen Leben teilnehmen zu können sowie Zugang zu Arbeitsplätzen und Dienstleistungen der täglichen Versorgung oder des Gesundheitswesens zu haben. Auf der anderen Seite führt die demografische Entwicklung auch dazu, dass viele Senioren bis ins hohe Alter mobil bleiben, indem sie den eigenen Pkw nutzen. Diese individuelle Mobilität wird noch durch weitere alternative Formen der Mobilität, wie bspw. Mitnahme-Verkehre von Familienmitgliedern, Nachbarn, Freunden, etc. ergänzt, sodass in vielen ländlichen Räumen eine Grundversorgung durch individuelle Mobilität gewährleistet wird.

Im Bereich des öffentlichen Verkehrs existieren ebenfalls gute Alternativen zu den starren und konventionellen (Bus-)Linienverkehren, die jedoch, anders als in urbanen Räumen, selten als tatsächliche Alternativen zu einer individuellen Mobilität wahrgenommen werden. Dies mag an mangelnder Sichtbarkeit resp. Zugänglichkeit oder an jahrelang aufgebauten Vorbehalten gegenüber dem öffentlichen Personennahverkehr (ÖPNV) begründet sein. Denn der ÖPNV in seiner konventionellen, liniengebundenen Form hat im ländlichen Raum mit pluralistischen Herausforderungen zu kämpfen: Einrichtungen der Daseinsvorsorge, wie bspw. Schulen, Einkaufsmöglichkeiten, Haus- und Fachärzte, konzentrieren sich oftmals nur in Orten einer höheren Zentralitätsstufe. Additiv hierzu existieren weitere, nicht zentralisierte Ziele, die seitens des ÖPNV bedient werden, wie bspw. Freizeitziele, abseits gelegene Industriegebiete oder größere Arbeitgeber. Die disperse Siedlungsstruktur im ländlichen Raum führt dazu, dass den zentralen Zielen von Mobilität eine Vielzahl an verstreuten Quellgebieten gegenübersteht. Die Problematik des ÖPNV resultiert daraus, dass er, ,anders als in großen Städten [...] kein Gegengewicht zum überbordenden motorisierten Individualverkehr (MIV) bildet, sondern vorrangig ein Instrument der Daseinsvorsorge ist" (Kirchhoff und Tsakarestos 2007, S. 1). Dementsprechend muss er die Diskrepanz zwischen Angebot (zentralisierte Ziele) und Nachfrage (Siedlungsgebiete als Quelle von Mobilität) überbrücken. Die Wechselwirkung zwischen ,abnehmender Siedlungsdichte und dem Rückzug von Einrichtungen der Daseinsvorsorge aus der Fläche führt zu einer Vergrößerung der Entfernung, die die Einwohner ländlicher Räume [...] überwinden müssen“ (Brenck et al. 2016, S. 20). Diese Divergenz erschwert die Generierung eines ÖPNV-Angebots, das alle Mobilitätswünsche gleichermaßen berücksichtigt. Insbesondere die abnehmende Siedlungsdichte hat letzten Endes die Konsequenz, dass innerhalb der Einzugsbereiche immer weniger potenzielle Fahrgäste wohnen.

Gleichermaßen trifft es zu, dass ein ÖPNV-Angebot noch so gut und passend auf die Mobilitätsbedürfnisse von Einwohnern einer ländlichen Region abgestimmt sein kann, entscheidend ist, wie dieses Angebot von der Bevölkerung wahrgenommen wird. Hierbei spielt bspw. die Gewohnheit eine große Rolle: Fährt man bereits seit Jahren mit dem eigenen Pkw, der für viele Einwohner ländlicher Räume die einzige und verlässlichste Mobilitätsoption darstellt, nimmt man zusätzliche Mobilitätsangebote kaum wahr resp. interessiert sich möglicherweise nicht dafür. Ähnlich verhält es sich mit der Nutzung eines ÖPNV-Angebots im ländlichen Raum. Die tatsächliche Auseinandersetzung mit der eigenen Mobilität findet so gut wie nicht statt, da das Mobilitätsverhalten von Gewohnheiten 
bestimmt wird. Lediglich bei Verspätungen, Unpünktlichkeit oder sonstigen Prozessen, die den reibungslosen Ablauf von Mobilität stören, wird über Mobilität nachgedacht und diese möglicherweise anders wahrgenommen.

Das Ziel des vorliegenden Beitrages ist es, die Mobilitätswahrnehmung der Bevölkerung eines ländlich geprägten Gebiets darzustellen. Um die Einstellung und Wahrnehmung der Einwohner hinsichtlich des ÖPNV und der Mobilität im Allgemeinen abzufragen, wurden 2015 alle Haushalte in der Gemeinde Bad Birnbach angeschrieben. Ziel dieser Befragung war es, aus den Mobilitätsbedürfnissen der Bevölkerung ein ÖPNVKonzept zu erarbeiten. In diesem Zusammenhang wurden 2015 ebenfalls alle Beherbergungsbetriebe in Bad Birnbach mit der Bitte angeschrieben, Aussagen über das Mobilitätsverhalten von Touristen vor Ort zu treffen. Beide Erhebungen wurden dazu benutzt, ein Mobilitätskonzept zu entwickeln, welches mit der Einführung des Rufbussystems 2016 realisiert wurde. Unabhängig davon wurde 2017 der autonome Shuttlebus in Bad Birnbach eingeführt. Um Unterschiede in der Mobilitätswahrnehmung und dem Mobilitätsverhalten von Bevölkerung und Touristen festzustellen, kam es 2018 erneut zu einer Befragung der Haushalte und Beherbergungsbetriebe. Unter Zuhilfenahme diverser Indikatoren, wie bspw. der „Bekanntheit der Verkehrsgemeinschaft Rottal-Inn“ oder dem „Rücklauf resp. der Teilnahme an der Befragung“, kann aus den beiden Erhebungen abgeleitet werden, inwieweit die Realisierung eines autonomen Shuttlebusses zu einer verbesserten Wahrnehmung des ÖPNV-Angebots beiträgt.

\subsection{Mobilitätsverhalten in ländlichen Räumen}

\subsubsection{Erläuterung zentraler Mobilitätsbegriffe}

Mobilität im Allgemeinen bedeutet ,Beweglichkeit von Personen, allgemein und als Möglichkeit“ (Becker et al. 1999, S. 71). Potenzielle Mobilität bezeichnet somit die Möglichkeit, sich bewegen zu können. Wohingegen realisierte Mobilität (=Verkehr) als eine ,realisierte Beweglichkeit, [zur] Befriedigung von Bedürfnissen durch Raumveränderung“ (Becker et al. 1999, S. 71) interpretiert werden kann. Entgegen der räumlichen Mobilität, die oftmals als Wanderungsbewegung ausgelegt wird, bezeichnet Mobilität in dem Kontext des vorliegenden Artikels die alltägliche Mobilität, die mit einem Verkehrsmittel erbracht wird. Verkehr ist somit ,die Ortsveränderung von Objekten (z. B. Güter, Personen, Nachrichten) in einem definierten System" (Ammoser und Hoppe 2006, S. 21).

In diesem Zusammenhang spielt das Mobilitätsverhalten der Bevölkerung eine große Rolle. An diesem Faktor kann abgelesen werden, wie Menschen ihre eigene Mobilität wahrnehmen und tatsächliche Mobilität zum Ausdruck bringen. Das Mobilitätsverhalten „hat direkte und indirekte Auswirkungen auf das Verkehrswesen, z. B. in Form des Verhaltens der Teilnehmer am Verkehr [... oder das] Nutzungsverhalten der Verkehrsteilnehmer bezüglich der ihnen zur Verfügung stehenden Verkehrsmittel“" (Ammoser und Hoppe 2006, S. 11). Das Mobilitätsverhalten spiegelt sich in unterschiedlichen Faktoren wider: 
Als plakativer Ausdruck des Mobilitätsverhaltens kann der Modal Split appliziert werden. Je nach Untersuchungsraum stellt dieser dar, welche Verkehrsmittel von den Verkehrsteilnehmern in einem bestimmten Zeitraum (bspw. innerhalb des letzten Jahres) oder zu einem bestimmten Zweck (bspw. zum Einkauf) genutzt wurden. Oftmals wird der Modal Split herangezogen, um zu belegen, wie nachhaltig sich eine Bevölkerungsgruppe bewegt (z. B. Wie hoch ist der Anteil der nachhaltigen Verkehre, wie Bahn, Bus, Rad und Fuß, am Gesamtverkehr?).

Einen entscheidenden Einfluss auf das Mobilitätsverhalten der Bevölkerung eines Raums hat die individuelle Einstellung zur Mobilität. Häufig wird bspw. aufgrund politischer oder ökologischer Werte auf die Nutzung eines eigenen Pkw verzichtet. Entsprechend der individuellen Überzeugung zur Mobilität und dem daraus resultierenden Verkehrsverhalten, bildet sich eine gewisse Art von Mobilitätswahrnehmung heraus, die für jeden Menschen unterschiedlich sein kann, auch wenn derselbe Weg zurückgelegt wird. Um Mobilitätswahrnehmung messen zu können, bedarf es unterschiedlicher Zugangsweisen zu der Vorstellung, inwieweit Mobilität bspw. passiv oder aktiv wahrgenommen wird. Die aktive Wahrnehmung von Mobilität betrifft den Verkehrsteilnehmer ganz unmittelbar und ohne dazwischen geschaltete Instanzen. Alleine durch die Teilnahme am Verkehr, sei es zu Fuß, per Pkw, Bus, Bahn oder Fahrrad, erfährt man Mobilität und nimmt diese direkt wahr. Hierdurch entstehen Emotionen, die mit Mobilität verknüpft werden. Bspw. nimmt man das Verkehrsmittel Bahn meistens dadurch wahr, dass die Nutzung dieses Verkehrsmittels des Öfteren mit verspäteten Ankünften am Zielort assoziiert und dementsprechend mit einer negativen Empfindung belegt wird. Demgegenüber steht die passive Wahrnehmung von Mobilität. Diese basiert zumeist auf Erzählungen oder Berichten Dritter. Neben Freunden und Familienmitgliedern sind insbesondere die Informationsmedien für unsere Wahrnehmung von Mobilität verantwortlich. Negative, wie auch positive, Berichterstattung über die Entwicklung des Fahrpreises im ÖPNV, die Verstopfung unserer Straßen durch zu viele Pkw oder auch über neue Formen der Mobilität prägen unsere Wahrnehmung von Verkehr und Mobilität, ohne dass wir aktiv an dieser teilhaben. Der vorliegende Beitrag definiert die Wahrnehmung von Mobilität etwas eingeschränkter. Der Fokus liegt insbesondere auf der Wahrnehmung und Nutzung des ÖPNV. Es soll erörtert werden, warum Einwohner ländlicher Räume den ÖPNV anders wahrnehmen als Bewohner eines urbanen Umfelds, bei denen der ÖPNV so selbstverständlich zur Mobilität dazu gehört wie der Pkw. Trotz der Beschränkung auf die Verkehrsmittel des ÖPNV wird die Wahrnehmung möglichst aller Verkehrsteilnehmer dargestellt, unabhängig davon, welches Verkehrsmittel von diesen genutzt wird.

Um grundsätzlich einen umfassenden Überblick über die Mobilitätswahrnehmung innerhalb eines Raumes zu erhalten, ist daher die direkte Nachfrage bei den Mobilitätsteilnehmern von entscheidender Bedeutung. Alleine aus der Interpretation des Modal Splits kann die Wahrnehmung nicht abgeleitet werden. Um die Wahrnehmung von Mobilität daher in der Gemeinde Bad Birnbach zu erheben, wurden zwei zeitlich versetzte Haushaltsbefragungen durchgeführt. Neben der Dokumentation ihrer aktiven Mobilität, in Form von Wegetagebüchern, wurden die Einwohner Bad Birnbachs darum gebeten, 
Fragen zum ÖPNV in Bad Birnbach zu beantworten, aus welchen sich Rückschlüsse auf die Wahrnehmung des ÖPNV in Bad Birnbach ziehen lassen. Ebenso sollten Beherbergungsbetriebe Auskunft über das Mobilitätsverhalten von Touristen geben.

\subsubsection{Neue Mobilitätsformen}

Wie es die Bezeichnung bereits vermuten lässt, ist der Mobilitätsmarkt ein Markt, der ständig in Bewegung ist. Eine Vielzahl technischer Neuerungen, zusammengefasst unter dem Begriff der Fahrassistenzsysteme, trägt bereits heutzutage zur Verkehrssicherheit bei und unterstützt den Fahrer im MIV bei der Durchführung seines Mobilitätsbedürfnisses. Neben fahrzeuginternen Neuerungen können ebenfalls Innovationen im Bereich der Antriebstechnik als neue Formen der Mobilität angesehen werden. In diesem Zusammenhang ist v. a. die E-Mobilität zu nennen, die auf konventionelle Antriebsarten (Diesel und Benzin) verzichtet. Eine flächenhafte Abdeckung mit E-Mobilität ist jedoch noch lange nicht in Sicht, da insbesondere die Ladeinfrastruktur noch weiter ausgebaut werden muss.

Viele Jahre galt der ÖPNV im ländlichen Raum als die einzige ernsthafte Alternative zum MIV. Dies ist auch in weiten Teilen ländlicher Räume noch heute so. Jedoch hat auch der ÖPNV in seiner konventionellen Form immer mehr mit Schwierigkeiten zu kämpfen, insbesondere wenn der Faktor Wirtschaftlichkeit betrachtet wird. Denn die abnehmende Bündelungsfähigkeit des ÖPNV in seiner konventionellen Form bewirkt einen zunehmend defizitären Betrieb. Wenn die Mobilitätsnachfrage über den ÖPNV im ländlichen Raum zu wirtschaftlichen Konditionen für Verkehrsunternehmen gedeckt werden soll, ist eine entsprechende Auslastung der Fahrzeuge erforderlich, sodass die Kosten durch Fahrgeldeinnahmen gedeckt werden können. Um jedoch hohe Auslastungsgrade zu erzielen, müssen aufgrund der geringen Bevölkerungsdichte viele Haltestellen angefahren und weite Distanzen zurückgelegt werden. Dies führt wiederum zu langen Reisezeiten, die zum einen die laufabhängigen Betriebskosten erhöhen und zum anderen die Attraktivität für die Fahrgäste senken. Denn der Zeitvorteil des MIV wird umso größer, je mehr die ÖPNVVerbindung aufgrund des Einsammelns der Fahrgäste in der Fläche von der direkten Verbindung zwischen Quelle und Ziel abweicht.

Eine Bündelung der Verkehrsnachfrage im ÖPNV wird zudem durch die räumliche und zeitliche Diversifizierung der Fahrtwünsche erschwert: Nicht alle Verkehrsströme finden ausgerichtet auf einen zentralen Ort hin statt, sondern möglicherweise müssen verschiedene Orte für verschiedene Erledigungen aufgesucht werden. Darüber hinaus besteht aufgrund unterschiedlicher Öffnungs- und Arbeitszeiten oder individueller Vorlieben der Wunsch nach zeitlich flexibler Mobilität. Eine Bündelung der vielfältigen Mobilitätsbedürfnisse kann bspw. durch ein ,abgestuftes Mobilitätskonzept mit Linienverkehren auf den Hauptrelationen und geeigneten Zubringerangeboten für die flächenhafte ErschlieBung“" (BMVI 2013, S. 18) erreicht werden. 
Eine gute Bündelungsfähigkeit lässt sich für den Bereich des Schülerverkehrs konstatieren, zumal Schüler ,,[i]n der Regel [...] in nachfrageschwachen ländlichen Räumen [...] keine Alternative bei der Verkehrsmittelwahl“" (BMVBS 2009, S. 52) besitzen. Daher ist das ÖPNV-Angebot im ländlichen Raum sehr stark auf den Schülerverkehr ausgerichtet. Die auf die Schüler ausgelegten Linienverkehre sind somit das Rückgrat des ÖPNVAngebots im ländlichen Raum, allerdings ein ,zunehmend schwaches Rückgrat in Anbetracht sinkender Schülerzahlen und abweichender Bedürfnisse der übrigen ÖPNV-Nutzer“ (Mante 2009, S. 11).

Der Schülerverkehr repräsentiert auch stets ein gewisses Grundangebot für potenzielle weitere Fahrgäste - jedoch mit zeitlich und räumlich sehr starken Restriktionen. Ergänzend hierzu existieren dort, wo ausreichend Nachfrage besteht, weitere ÖPNV-Angebote, die entweder im Linienverkehr oder bedarfsorientiert über Rufbusse, Anruf-SammelTaxen o. ä. bedient werden (BMVBS 2009, S. 29 ff.).

Der zu beobachtende Niedergang des ÖPNV im ländlichen Raum zwingt die Bevölkerung dazu, sich Alternativen zum einen zum ÖPNV und zum anderen zum eigenen Pkw zu suchen, insofern dies im ländlichen Raum überhaupt möglich ist. Dementsprechend muss das klassische Angebot von „Bussen und Bahnen [um weitere Mobilitätsoptionen, wie] Car-Sharing, Taxen, Fahrradverleihsysteme und andere Dienstleistungen“ (Ackermann 2013, S. 3) erweitert werden. Daher tragen neue Formen der Mobilität zu einer Verbreiterung des Angebotsspektrums bei und können bspw. dabei helfen, die Letzte Meile zwischen einer Haltestelle und dem Zielpunkt des Mobilitätsbedürfnisses zu schließen.

Eine mögliche Lösung zur Behebung dieser Problematik ist die flächenhafte Umsetzung des Sharing-Gedankens. Dabei wird „Car-Sharing [...] als eine organisierte Form der gemeinsamen Nutzung eines oder mehrerer Autos durch mehrere Nutzer verstanden. Sie setzt die Mitgliedschaft in einem Verein oder einer Genossenschaft oder den Abschluss eines Nutzungsvertrages [...] voraus. [Der Anbieter] erhält für seine Dienstleistungen Entgelte von den Nutzern“" (Loose et al. 2004, S. 19). Bisherige Feldversuche mit Car-SharingAngeboten im ländlichen Raum zeigen jedoch noch nicht die gewünschten Effekte (Verringerung des MIV-Anteils und Etablierung des Sharing-Gedankens auch im ländlichen Raum). Dementsprechend stellt die Nutzung eines Car-Sharing-Angebots derzeit noch keine wirkliche Alternative dar.

Eine in den letzten Jahren immer wieder diskutierte Ergänzung des Mobilitätsangebots wird durch das Schlagwort autonomer Shuttleverkehr beschrieben. Die Vorteile, den konventionellen ÖPNV mit automatisierten Angeboten zu ergänzen, liegen auf der Hand: Neben der spürbaren Reduzierung laufabhängiger Kosten (durch Einsparung des Fahrpersonals) eignen sich autonome Shuttlebusse insbesondere für die Schließung der Ersten/ Letzten Meile. Alleine die Größe eines autonomen Shuttlebusses eröffnet neue Möglichkeiten, die von konventionellen Bussen nicht umgesetzt werden können. Die Fahrt in einen engen Ortskern gestaltet sich bspw. mit einem großen Bus weitaus schwieriger, als mit einem autonomen Shuttlebus. Gleichermaßen von Vorteil ist die Tatsache, dass ein autonomer Shuttlebus quasi keine Pausen benötigt. Außer in der Zeit, in der der autonome Shuttlebus aufgeladen wird, ist ein durchgängiger Betrieb ohne Verzögerungen möglich. 
Somit kann der Einsatz eines autonomen Shuttlebusses ganz anders geplant und realisiert werden. Die Ergänzung des ÖPNV-Angebots um autonom agierende Busse bildet derzeit den Höhepunkt der technischen Entwicklung im ÖPNV-Sektor.

Es steht außer Frage, dass die Einführung des autonomen Shuttlebusses in Bad Birnbach zu einer erhöhten Mobilitätswahrnehmung der dortigen Bevölkerung geführt hat. Alleine durch die umfassende Berichterstattung in lokalen und regionalen Medien ist kein Einwohner darum herumgekommen, sich mit dem Thema Mobilität in irgendeiner Art und Weise auseinanderzusetzen. Inwieweit die Einführung eines autonomen Shuttlebusses tatsächlich dazu beiträgt, dass sich Menschen wieder mehr mit ihrer eigenen Mobilität auseinandersetzen, soll nun im Folgenden erörtert werden.

\subsection{Mobilität im Landkreis Rottal-Inn}

Der Landkreis Rottal-Inn ist in insgesamt 31 Gemeinden aufgeteilt. Rund 117.000 Menschen leben in dem im Süden des Regierungsbezirks Niederbayern gelegenen Landkreis, der als Tourismusregion vor allem bei Wanderern, Radfahrern und Naturliebhabern bekannt ist. Als das „ländliche Bad“ (Rottal-Inn 2019, k. A.), ist Bad Birnbach der Hauptanziehungsort für Touristen aus Deutschland und dem Ausland. Aus Sicht der Raumordnung weisen die Gemeinden Simbach a. Inn und Pfarrkirchen eine mittelzentrale Versorgungsfunktion für ihren Einzugsbereich auf. Daneben sind mit insgesamt acht Kleinzentren und zwei Unterzentren die Versorgungsräume über den gesamten Landkreis - einer der "streusiedlungsreichsten Landkreise Deutschlands“ (Rottal-Inn 2019, k. A.) - verteilt (Regierung von Niederbayern 2008, S. 3 f.). Trotz der hohen Zersiedlung verzeichnet der Landkreis Rottal-Inn insgesamt ein positives Wanderungssaldo.

Durch den Landkreis verlaufen zwei Bahnlinien. Die sogenannte Rottalbahn bietet den Gemeinden Massing, Eggenfelden, Hebertsfelden, Pfarrkirchen, Anzenkirchen und Bad Birnbach Anschluss nach Mühldorf und Passau. Die Gemeinde Simbach ist durch eine weitere Bahnlinie an Mühldorf angebunden. Seit September 1997 besteht im Landkreis durch den Zusammenschluss von acht Verkehrsunternehmen die Verkehrsgemeinschaft Rottal-Inn (VGRI). Die gemeinsame Verwaltung ermöglicht ein einheitliches Tarifangebot und die gegenseitige Anerkennung der Fahrausweise in allen Bussen der VGRI und der Bahn innerhalb des Landkreises.

In Bad Birnbach, der Gemeinde, die im Fokus der Betrachtung des vorliegenden Beitrags liegt, leben etwa 5700 Menschen (Bayerisches Landesamt für Statistik 2019 ). Die im Osten des Landkreises gelegene Gemeinde ist in insgesamt 85 Ortsteile gegliedert und verzeichnet seit vielen Jahren ein kontinuierliches Bevölkerungswachstum (ebd.). Die Mobilität in der Gemeinde ist vor allem durch den MIV geprägt. Etwa 30 Buslinien verbinden die Gemeinden des Landkreises. Auf das öffentliche Nahverkehrsangebot wird in Abschn. 4.2 genauer eingegangen (vgl. Kap. 4, Jürgens). Seit dem 9. April 2018 verkehrt zusätzlich zum klassischen Busverkehr auch der Ruf Bus Rottal-Inn (RuBi) im Landkreis. Insgesamt vier Rufbus-Linien ergänzen das Mobilitätsangebot in den Gemeinden 
Dietersburg, Egglham, Bad Birnbach, Bayerbach und Triftern. Der Bedarfsbus fährt nur nach vorheriger telefonischer Anmeldung (VGRI-Geschäftsstelle - RBO Niederlassung Süd 2019).

\subsection{Vergleich der Erhebungen der Jahre 2015 und 2018}

Im Rahmen eines Mobilitätskonzeptes für den Landkreis Rottal-Inn wurde Ende des Jahres 2015 eine Befragung aller Haushalte und Beherbergungsbetriebe in der Gemeinde Bad Birnbach durchgeführt mit dem Ziel, ein flexibles, an den Bedarf der Bürgerinnen und Bürger angepasstes Verkehrskonzept zu erarbeiten. Die Haushalte und Beherbergungsbetriebe erhielten per Post einen zweiseitigen Fragebogen inklusive frankiertem Rückumschlag, in dem sie ihre häufigsten Wege sowie weitere Informationen zu Ihrem Mobilitätsverhalten angeben konnten. Die Daten wurden im Anschluss erfasst und ausgewertet. Im Rahmen der Begleitforschung zum autonomen Shuttlebus in Bad Birnbach haben 2018 erneut Haushalts- und Beherbergungsbetriebsbefragungen stattgefunden. Die Kontaktaufnahme und der Rückversand erfolgten auf gleiche Weise. Um eine Vergleichbarkeit der Aussagen zu ermöglichen, stimmen die Fragebögen von 2015 und 2018 größtenteils hinsichtlich des Aufbaus und der Formulierung der Fragen überein. Unterschiede bestehen vor allem im Hinblick auf die beteiligten Projektpartner und die Intention der Projekte, in deren Rahmen die Befragungen erfolgt sind. Im Jahr 2015 wurden die Bürgerinnen und Bürger sowie die Touristen und Beherbergungsbetriebe mit dem Ziel befragt, ein angepasstes, bedarfsorientiertes, flexibles und an die Mobilitätsbedürfnisse der Landkreisbevölkerung angepasstes Mobilitätsangebot zu konzipieren und umzusetzen. Die Befragung wurde von der Gemeinde Bad Birnbach in Zusammenarbeit mit der Regionalbus Ostbayern GmbH durchgeführt. Im Rahmen des Forschungsprojektes im Jahr 2018 waren mehrere Projektpartner an der Konzeption und Durchführung der Befragung beteiligt. Mit und neben der Unterstützung der Gemeinde, waren die Universität Würzburg (Kap. 9, Rauh et al.), die Technische Hochschule Ingolstadt (Kap. 6 und 7, Wintersberger et al.) und die DB Regio Bus, Region Bayern an der Konzeption, Durchführung und Auswertung der Befragung beteiligt. Die Vielfalt an Fragestellungen der einzelnen Projektpartner führte zu einem größeren Umfang des Fragebogens im Jahr 2018. Neben Fragen zur Veränderung des Mobilitätsverhaltens, lag der Fokus der Befragung auch auf der Nutzung und Akzeptanz des autonomen Shuttles.

Im Zeitraum zwischen den beiden Erhebungsphasen erfolgten zwei Entwicklungen im Bereich Mobilität in Bad Birnbach, die an dieser Stelle nochmals hervorzuheben sind: Zum einen verkehrt seit Oktober 2017 zwischen dem Marktplatz und der Rottal-Terme in Bad Birnbach der autonome Shuttlebus. Zum anderen wurde im Anschluss an das bereits thematisierte Mobilitätskonzept des Landkreises ein Rufbussystem eingeführt. Die Veränderungen, auf die im nachfolgenden Kapitel eingegangen wird, können somit nicht immer klar einem der beiden Ereignisse bzw. allgemeinen Entwicklungstrends zugeschrieben werden, sondern stellen Folgewirkungen der ganzheitlichen Weiterentwicklung der 
Tab. 3.1 Vergleich der Befragungen 2015 und 2018. (Quelle: eigene Darstellung)

\begin{tabular}{|c|c|c|}
\hline & 2015 & 2018 \\
\hline \multicolumn{3}{|l|}{ Haushaltsbefragung } \\
\hline Untersuchungsgebiet & Bad Birnbach & Bad Birnbach \\
\hline Rücklauf (in \%) & $7,6 \%$ & $12,9 \%$ \\
\hline Rücklauf (absolut) & 235 & 396 \\
\hline Befragungszeitraum & Oktober 2015 & Juli/August 2018 \\
\hline \multicolumn{3}{|c|}{ Befragung Beherbergungsbetriebe } \\
\hline Untersuchungsgebiet & Bad Birnbach & Bad Birnbach \\
\hline Rücklauf (in \%) & $14,9 \%$ & $44,9 \%$ \\
\hline Rücklauf (absolut) & 27 & 31 \\
\hline Befragungszeitraum & Juli/August 2015 & Oktober 2018 \\
\hline
\end{tabular}

Mobilität in Bad Birnbach in diesem Zeitraum dar. In Tab. 3.1 werden zunächst die Befragungen von 2015 und 2018 hinsichtlich einiger statistischer Kennwerte zu Rücklauf und Zeitraum zusammenfassend gegenübergestellt.

\subsection{Veränderungen in der Mobilitätswahrnehmung}

Für die Analyse der Veränderungen im Mobilitätsverhalten und in der Wahrnehmung des Mobilitätsangebotes werden in der vorliegenden Arbeit drei Indikatoren herangezogen: Die Veränderung der Wahrnehmung kann einerseits durch eine Steigerung der Bekanntheit der ansässigen Verkehrsgemeinschaft VGRI gemessen werden. Auch die Einschätzung der Entfernung zur nächstgelegenen Bushaltestelle stellt einen Faktor dar, der Rückschlüsse auf die Wahrnehmung des Mobilitätsangebotes geben kann. Daneben kann auch der Rücklauf aus den Befragungen als Indikator für die Veränderung der Mobilitätswahrnehmung dienen.

\section{Indikator: Bekanntheit VGRI}

Im Jahr 2015 gaben nur $7 \%$ der befragten Beherbergungsbetriebe an, die Verkehrsgemeinschaft des Landkreises zu kennen. Im Laufe der vergangenen drei Jahre hat sich die Bekanntheit deutlich, genauer um das 8-fache erhöht und stieg auf über $50 \%$. Die Veränderung der Bekanntheit der VGRI deutet bereits darauf hin, dass die Thematik der öffentlichen Mobilität in den vergangenen Jahren eine deutlich höhere Präsenz im Alltag der Bewohnerinnen und Bewohner sowie in der öffentlichen Berichterstattung der Gemeinde erreicht hat. Die größere Bekanntheit der VGRI bei den Beherbergungsunternehmen kann zudem an einem gestiegenen Interesse von touristischer Seite an öffentlichem Nahverkehr stammen. Die Durchführung der ersten Umfrage 2015 hat mit Sicherheit ebenfalls zur höheren Bekanntheit der VGRI bei der zweiten Umfrage 2018 ihren Beitrag geleistet (vgl. Abb. 3.1). 


\section{Kennen Sie die VGRI?}

2015

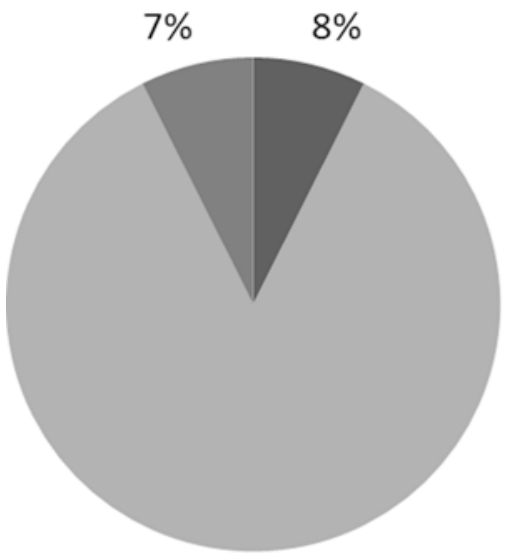

$85 \%$
Kennen Sie die VGRI?

2018

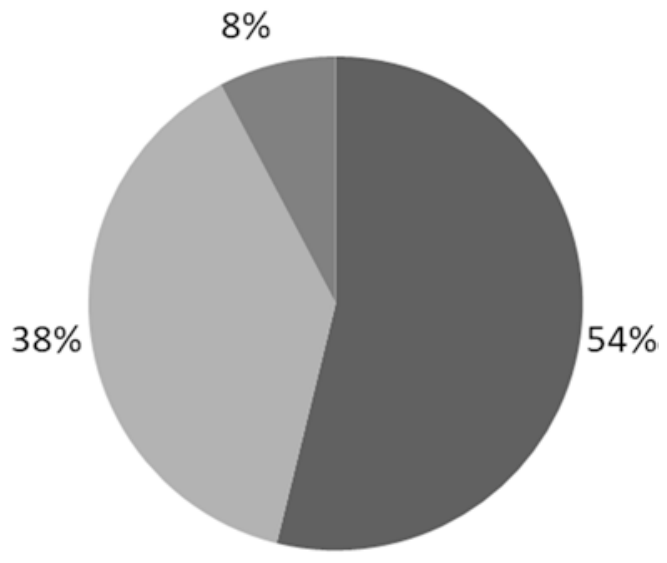

a Ja Nein Keine Angabe

- Ja Nein $\quad$ Keine Angabe

Abb. 3.1 Bekanntheit der VGRI (Verkehrsgemeinschaft Rottal-Inn). (Quelle: Ergebnis der Beherbergungsbetriebsbefragungen; eigene Darstellung)

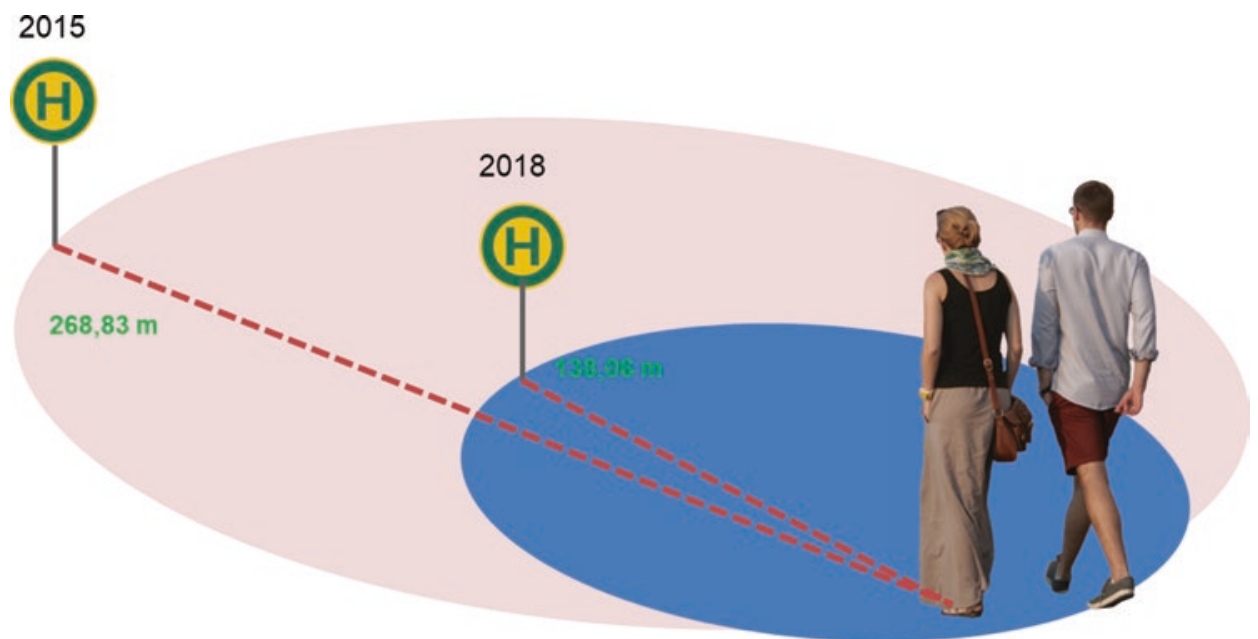

Abb. 3.2 Entfernung zur Haltestelle - Einschätzung. (Quelle: Ergebnis der Beherbergungsbetriebsbefragungen; eigene Darstellung)

\section{Indikator: Entfernung zur Bushaltestelle}

Einen weiteren Indikator für eine veränderte Wahrnehmung der Mobilitätsoptionen stellt die Einschätzung der Entfernung zur nächstgelegenen Bushaltestelle dar. Wie Abb. 3.2 
zeigt, hat sich die geschätzte Entfernung von 2015 auf 2018 halbiert, von 269 auf 138 Meter.

Der deutlich niedrigere Wert spricht dafür, dass die Barrieren zur Nutzung des öffentlichen Mobilitätsangebots - in diesem Fall die Entfernung zur Haltestelle als erste Hürde bereits geringer eingestuft werden. Da die Haltestellenanzahl in diesem Zeitraum nicht verdoppelt wurde, hat dieser Indikator eine sehr praxisnahe, wenn auch nur subjektiv empfundene Aussagekraft. Die niedrigere Einschätzung des Entfernungswertes deutet auf eine aufmerksamere und wahrscheinlich praktisch häufigere Wahrnehmung der öffentlichen Mobilitätsoptionen hin.

\section{Indikator: Rücklauf aus Befragungen}

Auch der hohe Rücklauf der Befragung des Jahres 2018 deutet auf ein höheres Interesse an Mobilitätsfragen im Landkreis hin. Auch wenn sich die Rahmenbedingungen der Haushaltsbefragung, wie unter Abschn. 3.4. ausgeführt, hinsichtlich des Umfangs und der beteiligten Umfragepartner unterscheiden, lässt sich der höhere Rücklauf nicht alleine auf diese zurückführen. Gerade durch den großen Umfang des Fragebogens, der 2018 an die Haushalte gerichtet war, wurde eine eher zurückhaltende Resonanz der Bürgerinnen und Bürger erwartet. Die Fragebögen, die in beiden Jahren an die Beherbergungsbetriebe gerichtet waren, unterschieden sich hinsichtlich Aufbau und Umfang nur gering. Dennoch war auch hier eine deutlich höhere Beteiligung im Jahr 2018 festzustellen.

Bei den Haushaltsbefragungen ist der Rücklauf von 7,6 \% auf 12,9\% (Tab. 3.1) gestiegen. Im Jahr 2018 haben 44,9\% der Beherbergungsbetriebe an der Befragung teilgenommen, im Jahr 2015 waren es lediglich 14,9\%.

Der hohe und im Vergleich zu 2015 deutlich gestiegene Rücklauf deutet auf ein wachsendes Interesse der Bürgerinnen und Bürger an Mobilitätsfragen hin. Dies kann unter anderem auf das ausgeweitete Angebot und die Aktivitäten zur Verbesserung der Mobilität im Landkreis und in der Gemeinde Bad Birnbach zurückzuführen sein, die auch von der Bevölkerung wahrgenommen werden. Auch die Entwicklungen und Neuerungen im Bereich Mobilität außerhalb der eigenen Region tragen dazu bei, die Bürgerinnen und Bürger für die Möglichkeiten einer modernen Mobilitätsplanung zu sensibilisieren und neugierig zu machen.

Anhand der drei Indikatoren - Bekanntheit der VGRI, Einschätzung der Entfernung zur nächstgelegenen Haltestelle und Rücklauf aus Befragungen - lassen sich Anhaltspunkte erkennen, die auf eine gestiegene Wahrnehmung der Mobilitätsmöglichkeiten in der Gemeinde schließen lassen. Dabei setzen die Indikatoren an unterschiedlichen Aspekten der Wahrnehmung an. Die Bekanntheit der VGRI als Indikator zeigt, dass die höhere Bedeutung von Mobilität im Landkreis auch indirekt stärker wahrgenommen wird. Die hohe Beteiligung an den Erhebungen deutet klar auf ein großes Interesse der Bevölkerung an der Thematik hin. Die niedrigere Einschätzung der Haltestellenentfernung stellt wiederum eine in dem Kontext dritte Dimension der Mobilitätswahrnehmung dar, da diese auf eine geringere Wirkung als Barriere zur Nutzung des öffentlichen Nahverkehrs hinweist. 
Die positiven Entwicklungen im Mobilitätsangebot der Gemeinde Bad Birnbach spiegeln sich folglich auch in den drei untersuchten Indikatoren zur Wahrnehmung der Mobilität wider. Sie lassen den Schluss zu, dass sowohl die Aufmerksamkeit und das Interesse der Bürgerinnen und Bürger für Mobilitätsfragen gestiegen sind (Indikatoren eins und zwei), als auch die tatsächliche Wahrnehmung i. S. v. Nutzung (Indikator drei) eine zunehmend wichtigere Rolle spielt.

\subsection{Fazit}

Veränderungen in der Mobilitätswahrnehmung, die sich zum Beispiel in der Bekanntheit der Verkehrsgemeinschaft oder einem höheren Interesse an Mobilitätsfragen äußern, stellen eine bedeutende Voraussetzung für eine Anpassung der Verhaltensmuster dar. Die vergleichsweise geringen Veränderungen in der Verkehrsmittelwahl, die sich aus den Haushaltsfragebögen ableiten lassen, verdeutlichen, dass eine Anpassung des Mobilitätsverhaltens eines längeren Zeitraums bedarf. Dementsprechend wäre eine weiterführende Untersuchung der Mobilität in Bad Birnbach durchaus sinnvoll. Nur so lässt sich mit Gewissheit sagen, dass sich die Mobilitätswahrnehmung durch die Integration des autonomen Shuttlebusses in die ÖPNV-Angebotsstruktur auch tatsächlich geändert hat, bspw. indem sich der Anteil des MIV am Gesamtverkehr in Bad Birnbach verringert oder indem sich die Nachfrage nach der Leistung des autonomen Shuttlebusses derart steigert, dass weitere Kapazitäten für autonome Strecken(abschnitte) geplant werden müssen. Ergänzend zu weiteren Haushaltsbefragungen in den Folgejahren, könnten auch qualitative Interviews stattfinden, um die tatsächliche Einstellung der Einwohner Bad Birnbachs zu autonomen Shuttlebussen zu erfahren.

Ob die Veränderung der Mobilitätswahrnehmung, die sich aus der vergleichenden Darstellung der Befragungen ableiten lässt, auch tatsächlich auf die Einführung eines autonomen Shuttlebusses zurückzuführen ist, bleibt also zunächst zweifelhaft. Da sich der verkehrliche Mehrwert des autonomen Shuttlebusses überwiegend für Touristen ergibt (Fahrt von der Therme zum Marktplatz), ist davon auszugehen, dass sich das Mobilitätsverhalten der Einwohner Bad Birnbachs durch die Einführung des autonomen Shuttlebusses nicht grundlegend geändert hat. Der Einsatz einer neuartigen Technik trägt jedoch zweifelsohne dazu bei, dass sich Menschen intensiver mit ihrer Mobilität und ihrem Mobilitätsverhalten auseinandersetzen. Hier hat die stetige mediale Berichterstattung über die Einführung des autonomen Shuttlebusses und dessen anschließender Entwicklung (bspw. Berichterstattung über den 10.000sten Fahrgast, etc.) einen großen Anteil daran, dass das Thema Mobilität resp. Öffentlicher Verkehr in den Fokus der Bevölkerung gerückt wurde. Letzten Endes können die hier erlangten Erkenntnisse daher nicht auf vergleichbare Räume übertragen werden. Für die Wahrnehmung von Mobilität spielen zu viele Faktoren eine Rolle, als dass mit Gewissheit gesagt werden kann, dass der autonome Shuttlebus einen nachweisbaren Beitrag dazu leistet. 


\section{Literatur}

Ackermann T (2013) Der ÖPNV. Rückgrat und Motor eines zukunftsorientierten Mobilitätsverbundes. Selbstverlag des VDV, Köln

Ammoser H, Hoppe M (2006) Glossar Verkehrswesen und Verkehrswissenschaften. Definitionen und Erläuterungen zu Begriffen des Transport- und Nachrichtenwesens, Nr. 2/2006 Diskussionsbeiträge aus dem Institut für Wirtschaft und Verkehr. Selbstverlag, Dresden

Becker U, Gerike R, Völlings A (1999) Gesellschaftliche Ziele von und für Verkehr. Heft 1 der Schriftenreihe des Instituts für Verkehr und Umwelt e. V. (DIVU). Dresden

BMEL - Bundesministerium für Ernährung und Landwirtschaft (2016) Bericht der Bundesregierung zur Entwicklung der ländlichen Räume 2016. Selbstverlag, Berlin

BMVBS - Bundesministerium für Verkehr, Bau und Stadtentwicklung (2009) Handbuch zur Planung flexibler Bedienungsformen im ÖPNV. Ein Beitrag zur Sicherung der Daseinsvorsorge in nachfrageschwachen Räumen. VisLab Wuppertal Institut, Bonn

BMVI - Bundesministerium für Verkehr und digitale Infrastruktur (2013) Langfristige Sicherung von Versorgung und Mobilität in ländlichen Räumen. Demografische Herausforderungen, interkommunale Kooperationen und Mobilitätsstrategien am Beispiel Nordfriesland. Selbstverlag, Berlin

Brenck A, Gipp C, Nienaber P (2016) Mobilität sichert Entwicklung. Herausforderungen für den ländlichen Raum. https://www.adac.de/_mmm/pdf/fi_mobilitaet\%20sichert_entwicklung_studie_0316_259064.pdf. (14.01.2019)

Kirchhoff P, Tsakarestos A (2007) Planung des ÖPNV in ländlichen Räumen. Ziele, Entwurf, Realisierung. B.G. Teubner Verlag/GWV Fachverlage GmbH, Wiesbaden

Landesamt für Statistik (2019): Bevölkerungsstand in den Gemeinden Bayerns.

Loose W, Mohr M, Nobis C (2004) Bestandsaufnahme und Möglichkeiten der Weiterentwicklung von Car-Sharing. Schlussbericht. Selbstverlag, Berlin

Mante J (2009) Mobilität im ländlichen Raum. Viele Wege, doch welches Ziel? Bundesanstalt für Landwirtschaft und Ernährung LandInForm. Magazin für ländliche Räume 10-11

Regierung von Niederbayern (2008): Fortschreibung des Regionalplans Landshut. http://www.regierung.niederbayern.bayern.de/media/aufgabenbereiche/2/raumordnung/rpla_b9_180108.pdf (06.02.2019)

VGRI-Geschäftsstelle - RBO Niederlassung Süd (2019) Wir fahren, wenn Sie anrufen! - Die Rufbuslinien Landkreis Rottal-Inn. http://www.vgrottal-inn.de/rufbus/ (07.02.2019)

Open Access Dieses Kapitel wird unter der Creative Commons Namensnennung 4.0 International Lizenz (http://creativecommons.org/licenses/by/4.0/deed.de) veröffentlicht, welche die Nutzung, Vervielfältigung, Bearbeitung, Verbreitung und Wiedergabe in jeglichem Medium und Format erlaubt, sofern Sie den/die ursprünglichen Autor(en) und die Quelle ordnungsgemäß nennen, einen Link zur Creative Commons Lizenz beifügen und angeben, ob Änderungen vorgenommen wurden.

Die in diesem Kapitel enthaltenen Bilder und sonstiges Drittmaterial unterliegen ebenfalls der genannten Creative Commons Lizenz, sofern sich aus der Abbildungslegende nichts anderes ergibt. Sofern das betreffende Material nicht unter der genannten Creative Commons Lizenz steht und die betreffende Handlung nicht nach gesetzlichen Vorschriften erlaubt ist, ist für die oben aufgeführten Weiterverwendungen des Materials die Einwilligung des jeweiligen Rechteinhabers einzuholen.

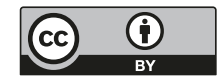

